\title{
PERFIL EPIDEMIOLÓGICO DA MORTALIDADE NEONATAL NO ESTADO DO PIAUÍ, BRASIL
}

Epidemiological profile of neonatal mortality in the state of Piaui, Brazil

Perfil epidemiológico de la mortalidad neonatal en el estado de Piauí, Brasil

Amanda Costa Pinheiro - Enfermeira - Assessora técnica do Conselho de Secretarias Municipais do Piauí • E-mail: amanda@cosemspi.org.br

Samuel Chaves Cardoso de Matos • Acadêmico de Medicina da Universidade Federal do Piauí-UFPI • E-mail: samuel_np@hotmail.com

Zenira Martins Silva • Assistente Social • Mestre em Saúde Pública pela FIOCRUZ - Coordenadora de epidemiologia da Secretaria de Estado da Saúde do Piauí • E-mail: zeniramartins@hotmail.com

Lis Cardoso Medeiros • Cirurgiã-dentista • Professora Titular de Biofísica da Universidade Federal do Piauí • E-mail: lismarinho10@gmail.com

Autora responsável pela correspondencia:

Amanda Costa Pinheiro • E-mail: amanda@cosemspi.org.br 


\section{ciêncíncia
pural}

\section{RESUMO}

Introdução: A redução da mortalidade infantil é ainda um desafio para os serviços de saúde. Apesar do declínio observado no Brasil, a mortalidade infantil permanece como uma grande preocupação em Saúde Pública. No Piauí com a implantação da Rede Cegonha e do Componente Neonatal nos últimos anos, pode-se perceber um declínio no índice de mortalidade materna, infantil e neonatal, mas ainda continua se perdendo mães e bebês por causas evitáveis. Apesar dos avanços que o estado teve após a implantação da Rede Cegonha esse desenvolvimento não se deu por igual em todas as regiões de saúde, ficando a maior parte da assistência centralizada ainda na capital. Objetivo: Este trabalho teve o objetivo de avaliar o perfil epidemiológico da mortalidade neonatal no estado do Piauí. Metodologia: Foi realizado um estudo retrospectivo, descritivo de abordagem quantitativa, cujos dados foram coletados no sistema do DATASUS e tabulados utilizando o TABWIN E TABNET. Resultados: Observou-se uma redução do número absoluto total de óbitos entre os anos 2007 a 2015, havendo um aumento de aproximadamente 4,5\% entre os anos de 2015 e 2016. A mortalidade neonatal precoce compreendeu mais de $3 / 4$ dos óbitos neonatais. Mais de $53 \%$ dos óbitos são de recém nascido de baixo peso. Cerca de $48,7 \%$ dos óbitos referem-se aos prematuros. Conclusões: Os achados revelaram as principais características dos óbitos neonatais no Piauí na série temporal (2007-2016).

Palavras-Chave: Mortalidade Infantil; Saúde da Mulher; Saúde Pública; Qualidade de Vida.

\section{ABSTRACT}

Introduction: The reduction of infant mortality is still a challenge for health services. Despite the decline observed in Brazil, infant mortality remains a major concern in Public Health. In Piauí with the implementation of the Cegonha Network and the Neonatal Component in recent years, a decline in the maternal, infant and neonatal mortality rate can be noticed, but mothers and babies are still being lost due to preventive causes. Despite the advances that the state had after the implementation of the stork network, this development did not happen equally in all health regions, with most of the centralized assistance still in the capital. Objective: This study aimed to assess the epidemiological profile of neonatal mortality in the state of Piauí. Methodology: A retrospective, descriptive study with a quantitative approach was carried out, and the data were collected in the DATASUS system and tabulated using TABWIN AND TABNET. Results: There was a reduction in the total absolute number of deaths between the years 2007 to 2015, with an increase of approximately $4.5 \%$ between the years 2015 and 2016. Early neonatal mortality comprised more than 3/4 of neonatal deaths . Over $53 \%$ of deaths are of low birth weight newborns. About $48.7 \%$ deaths refer to premature infants. Conclusions: The findings revealed the main aracteristics of neonatal deaths in Piauí in the time series (2007-2016).

ywords: Child mortality; Women's Health; Public health; Quality of life. 


\section{RESUMEN}

Introducción: la reducción de la mortalidad infantil sigue siendo un desafío para los servicios de salud. A pesar de la disminución observada en Brasil, la mortalidad infantil sigue siendo una preocupación importante en la salud pública. En Piauí con la implementación de la Red Cegonha y el Componente Neonatal en los últimos años, se puede notar una disminución en la tasa de mortalidad materna, infantil y neonatal, pero las madres y los bebés todavía se están perdiendo debido a causas preventivas. A pesar de los avances que tuvo el estado después de la implementación de la red de cigüeñas, este desarrollo no ocurrió por igual en todas las regiones de salud, con la mayor parte de la asistencia centralizada todavía en la capital. Objetivo: Este estudio tuvo como objetivo evaluar el perfil epidemiológico de la mortalidad neonatal en el estado de Piauí. Metodología: se realizó un estudio descriptivo retrospectivo con un enfoque cuantitativo, y los datos se recopilaron en el sistema DATASUS y se tabularon con TABWIN Y TABNET. Resultados: Hubo una reducción en el número absoluto total de muertes entre los años 2007 a 2015, con un aumento de aproximadamente 4.5\% entre los años 2015 y 2016. La mortalidad neonatal temprana comprendió más de 3/4 de las muertes neonatales. . Más del 53\% de las muertes son de recién nacidos con bajo peso al nacer. Alrededor del $48.7 \%$ de las muertes se refieren a bebés prematuros. Conclusiones: Los hallazgos revelaron las principales características de las muertes neonatales en Piauí en la serie temporal (2007-2016).

Palabras clave: Mortalidad infantil; La salud de la mujer; Salud pública; Calidad de vida. 


\section{Introdução}

A redução da mortalidade infantil é ainda um desafio para os serviços de saúde. Faz parte das Metas do Desenvolvimento do Milênio, compromisso assumido pelos países integrantes da Organização das Nações Unidas (ONU), do qual o Brasil é signatário, para o combate à pobreza, à fome, às doenças, ao analfabetismo, à degradação do meio ambiente e à discriminação contra a mulher, visando ao alcance de patamares mais dignos de vida para a população, uma vez que a mortalidade infantil reflete as condições de vida da sociedade ${ }^{1}$.

A assistência pré-natal, ao parto e puerpério têm sido defendida amplamente por possibilitar a redução da morbimortalidade. Sua importância está evidenciada por fazer parte das pautas internacionais, como exemplo, os objetivos estabelecidos em 2000 pós reunião da Cúpula do Milênio das Nações Unidas, constava a redução da Razão de Mortalidade Materna (RMM) para valores iguais ou inferiores a 35/100.000 nascidos vivos até 2015. Tal objetivo foi traçado mediante estudos da época em que apresentavam uma curva acentuadamente decrescente, entretanto o que se observou posteriormente, foi que estes valores começaram a se estabilizar. O objetivo proposto não foi alcançado, porém a meta se fez importante para incentivar o governo a criar estratégias. Visto a estabilidade da curva de redução da RMM a nova meta proposta pela Agenda 2030, em seu subitem 3.1 é que, em 2030 haja redução global da RMM para valores inferiores a 70/100.000 nascidos vivos e em seu sub item 3.2 consta reduzir a mortalidade neonatal para 12/1.000 nascidos vivos ${ }^{2}$.

No Brasil, segundo preconizado pelo Ministério da Saúde (MS), a assistência pré-natal e pós-natal tem o intuito de detectar e intervir precocemente em morbidades que possam interferir no desenvolvimento fetal adequado e que possa resultar em agravos e levar ao óbito tanto da mãe como do feto, ou recém-nascido. Para que a intervenção seja satisfatória é necessário um sistema hospitalar ágil e bem articulado atenção primária, além de qualificação assistencial apropriada ${ }^{3}$. Segundo o manual cnico do MS 4 , deve haver a garantia da atenção no pré-natal de forma humanizada, seja, deve haver a valorização dos sujeitos envolvidos no processo de produção da de além do atendimento ao outro como sujeito. Autores ${ }^{5}$ fazem crítica a 
medicalização no cuidado a gestante e aponta para as beneficies dos modelos desmedicalizados em que incluiria modelos humanísticos. Ainda para este autor, há problemas de interlocução entre equipes de saúde generalistas e especializadas em obstetrícia o que pode diminuir a qualidade da assistência.

É possível avaliar o processo e qualidade assistencial pré-natal por diversas vias: pesquisa de prontuários; entrevistas; indicadores de mortalidade materna e infantil, os quais refletiriam o resultado grosseiro do processo, tais metodologias têm sido utilizadas em diferentes estudos 6-9.

Apesar do declínio observado no Brasil, a mortalidade infantil permanece como uma grande preocupação em Saúde Pública. Os níveis atuais são considerados elevados e incompatíveis com o desenvolvimento do país, havendo sérios problemas a superar, como as persistentes e notórias desigualdades regionais e intra-urbanas, com concentração dos óbitos na população mais pobre, além das iniquidades relacionadas a grupos sociais específicos ${ }^{10}$.

Autores ${ }^{11}$ avaliam dimensões relacionadas a acesso (infra-estrutura, ações gerenciais para garantir o acesso) e qualidade do cuidado (ações gerenciais e clínicas para a qualidade do cuidado) das regiões brasileiras concluindo, também, que os piores indicadores estão distribuídos nas regiões norte e nordeste.

No Piauí com a implantação da Rede Cegonha e do Componente Neonatal nos últimos anos pode-se perceber um declínio no índice de mortalidade materna, infantil e neonatal, mas ainda continua-se perdendo mães e bebês por causas evitáveis. Por deficiência ou na assistência ao parto e ao recém-nascido (RN), ou por um pré-natal de baixa qualidade. Somente no ano de 2015 tivemos um número de 732 óbitos infantis sendo: neonatal precoce 414 óbitos; neonatal tardio 120 óbitos; pós-neonatal 198 óbitos segundo o relatório do Comitê de Mortalidade Infantil e Fetal do Estado do Piauí e dados do painel de monitoramento da SES/PI.

Entende-se por mortalidade infantil os óbitos ocorridos entre os 0 (zero) e 1 (um) ○ de idade. Este indicador também está presente na Agenda 30 devido a sua portância por refletir condições de saneamento, de saúde, educação e distribuição enda. 
O Brasil tem avançado muito nos últimos anos na melhoria deste indicador e pode estar relacionado as políticas de distribuição de renda (como o programa Bolsa Família) e a organização da saúde em unidades de Estratégia Saúde da Família. Estes autores fazem um estudo da expansão das políticas públicas e da variação dos indicadores ao longo dos anos, observando uma relação entre eles. Em seguida fazem projeções para o futuro em cenários diferentes, considerando a continuação dos avanços nas políticas públicas, em que os indicadores tenderiam a melhorarem constantemente, e um segundo cenário, com cortes e redução da diminuição das desigualdades sociais, que acarretaria piora do bem-estar social e, consequentemente, piora dos indicadores de moralidade infantil ${ }^{12}$. Outro trabalho ${ }^{13}$ já havia relatado a proporção inversa entre a mortalidade infantil e a expansão da cobertura populacional pela Estratégia Saúde da Família.

A Agenda 30, coloca como objetivo que as nações alcancem uma mortalidade infantil (considerado na Agenda 30 óbito de menores de 5 anos) menor que 25 óbitos por 1.000 nascidos vivos. Segundo França et al (2017) ${ }^{16}$, em 1990 o Brasil registrou 195.506 óbitos entre 0-5 anos (cerca de 61 óbitos por 1.000 nascidos vivos) e estes números melhoraram bastante em 25 anos alcançando, em 2015, 16 óbitos por 1.000 nascidos vivos (43.226, em números absolutos), colocando o Brasil dentro da meta da Agenda 30. Segundo trabalho de 2016 ${ }^{14}$, o Brasil alcançou os objetivos da ODS 3 ainda em 2011.

Uma subdivisão importante, dentro da mortalidade infantil, é a mortalidade neonatal, compreendida como os óbitos ocorridos nos primeiros 27 dias de vida, por refletir principalmente as condições da assistência pré-natal, natal e pós-natal.

Desde a década de 90 a mortalidade neonatal é o principal componente da mortalidade infantil. Cerda de $25 \%$ da mortalidade infantil ocorre nas primeiras $24 \mathrm{~h}$ de vida $^{15}$. Segundo outra pesquisa ${ }^{16}$, em 2015, 65,4\% da mortalidade infantil compreendia a mortalidade neonatal, o que significou um aumento do percentual bservado em 1990 que era de 41,7\%. Este aumento pode ser explicado pelos dados resentados por estes autores que demonstram um aumento de óbitos de prematuros 
e malformação congênita e uma redução significativa das mortes por diarréia, por exemplo.

Os dados epidemiológicos são importantes meios de direcionamento das políticas públicas. Neste sentido o SUS utiliza ferramenta de captação de informações, dentre elas o Sistema de Informação sobre Mortalidade (SIM) e o Sistema de Informação de Nascidos Vivos (SINASC). Ambos sistemas subsidiam as informações necessárias para elaboração de estratégias pelo Estado.

O Sistema de Informação sobre Mortalidade foi criado pelo Departamento de Informática do Sistema Único de Saúde (DATASUS) e objetiva captar informações sobre a mortalidade do país. Um dos principais meios para captação da informação utilizada pelo SIM é a Declaração de Óbito (DO). Para que os dados disponíveis no sistema reflitam a realidade é necessário a fidedignidade no preenchimento, bem como o preenchimento completo da declaração de maneira adequada. Autores ${ }^{16}$ analisaram a precariedade e incompletitude de dados do SIM em alguns municípios concluindo que o déficit mais importante dos dados se devia ao componente humano, ou seja, ao preenchimento inadequado da DO e do SIM. Em outra pesquisa ${ }^{17}$, realizando um estudo similar em um hospital universitário encontrou o mesmo problema, neste caso mais da metade das DO estavam incompletas.

Cabe ressaltar a importância epidemiológica dos dados fornecidos pelo SIM ${ }^{17}$. Ao mesmo tempo, visto o componente deficitário no preenchimento adequado da $\mathrm{DO}$, deve-se atentar à possibilidade de subnotificações. Ainda assim, os dados do SIM podem ser considerados em estudos da situação de saúde.

O SINASC é outro sistema importante utilizado pelo Sistema Único de Saúde (SUS) para verificação das condições do pré-natal e do parto. A principal ferramenta do SINASC é a Declaração de Nascido Vivo (DN), documento emitido após o parto constando uma série de informações relacionadas a condição da gravidez, do recémascido e da mãe.

Os dados do SINASC sofreram críticas, assim como os do SIM, devido o enchimento ser afetado pelo componente humano na geração da informação. balhos recentes mostram que, apesar de haverem problemas no sistema, os dados 
são satisfatórios, há boa adesão e portanto, pode-se considerar que há boa confiabilidade dos dados ${ }^{18,19}$.

Consideram-se óbitos infantis evitáveis aqueles que por ações efetivas dos serviços de saúde poderiam ter sido redutíveis. Esses óbitos também são considerados como eventos sentinelas da qualidade da assistência à saúde e o seu acontecimento indica falha na atenção à saúde ${ }^{20}$.

O coeficiente de mortalidade infantil é importante indicador das condições de saúde da população, muito utilizado no planejamento e avaliação das políticas de saúde. O componente neonatal da mortalidade infantil concentra a maior parcela dos óbitos infantis e está associado às condições de assistência ao pré-natal e ao parto. A relevância de se estudar as circunstâncias dos óbitos infantis deve-se ao fato de serem mortes precoces e potencialmente evitáveis pelo acesso em tempo oportuno a serviços qualificados de saúde ${ }^{21}$.

A mortalidade neonatal está vinculada a causas preveníeis, relacionadas ao acesso e a utilização dos serviços de saúde, e a qualidade da assistência pré-natal, ao parto e ao recém-nascido. Com objetivo de diminuir a mortalidade materno-infantil institui-se em 2004, a Política Nacional de Atenção Integral a Saúde da Mulher, cujo objetivo é promover a melhoria das condições de vida e saúde das mulheres por meio da: (i) garantia de direitos; e (ii) ampliação do acesso aos meios e serviços de promoção, prevenção, assistência e recuperação da saúde.

A Rede Cegonha (RC) foi instituída no âmbito do Sistema Único de Saúde, por meio da portaria 1.459, de 24 de junho de 2011. Dentre seus objetivos estão a implantação de um novo modelo de atenção ao parto, ao nascimento, ao crescimento e ao desenvolvimento da criança de zero aos vinte e quatro meses e o da redução da mortalidade materna e infantil com ênfase no componente neonatal.

Apesar dos avanços que o estado teve após a implantação da rede cegonha esse senvolvimento não se deu por igual em todas as regiões de saúde, ficando a maior te da assistência centralizada ainda na capital.

Segundo o Decreto 7508, de 2011 regulamenta a organização em região de saúde paço geográfico contínuo constituído por agrupamentos de Municípios limítrofes, 


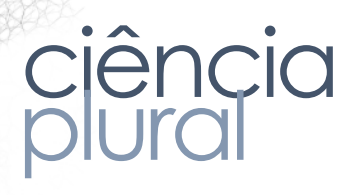

delimitado a partir de identidades culturais, econômicas e sociais e de redes de comunicação e infraestrutura de transportes compartilhados, com a finalidade de integrar a organização, o planejamento e a execução de ações e serviços de saúde. No estado do Piauí, a Lei Complementar No 87 de 22 de agosto de 2007 institui as regiões de saúde no Estado em 28 aglomerados e 11 Territórios de Desenvolvimento (TD). Este trabalho avaliou o perfil epidemiológico da mortalidade neonatal no estado do Piauí.

\section{Metodologia}

Foi realizado um estudo retrospectivo, descritivo, abordagem quantitativa, e os dados foram coletados no sistema do DATASUS, utilizando o TABWIN E TABNET. A consolidação dos dados foi feita por estatística simples. O período utilizado para coleta dos dados foi de 2007 a 2016. Para atingir o objetivo proposto, foram analisadas as frequências absolutas das mortes infantis por residência e ocorrência; as proporções de óbitos neonatais em relação ao total de mortes infantis no ano e no período considerado; as proporções das mortes neonatais precoces e neonatais tardias; a mortalidade infantil em relação ao peso ao nascer; idade gestacional; os municípios com os maiores números absolutos de óbito infantil e neonatal e; os principais grupos de causas de óbitos infantis e neonatal.

A pesquisa foi submetida para apreciação do Comitê de Ética e Pesquisa da Universidade Federal do Piauí e seguiu os princípios éticos que constam na Resolução n ${ }^{o}$ 466, de 2012, do Conselho Nacional de Saúde tendo sido aprovada mediante o parecer $n^{0} 2.552 .399$.

\section{Resultados e Discussão}

O número total de óbitos, para o período compreendido entre os anos de 2007 2016, por residência divergiu em 1.000 (mil) em número de óbitos por ocorrência, hdo os valores de 8.415 (oito mil, quatrocentos e quinze) e 9.415 (nove mil, uatrocentos e quinze) óbitos, respectivamente. Tal informação pode estar relacionado incorreto abastecimento do sistema com informações. Outra hipótese seria a falta hotificação dos óbitos em que a residência da mãe foi ignorada. A distribuição entre 
os Territórios de Desenvolvimento (TD), demonstra uma concentração do número de óbitos no Território Entre Rios (gráfico 1) que compreende a capital do estado e suas cidades vizinhas, fato justificável pela maior densidade demográfica local e ainda pela disposição de centros especializados de atendimento ao período perinatal como a Maternidade Dona Evangelina Rosa. É notória a concentração dos óbitos nas TD que possuem as cidades mais populosas do estado como as regiões Planície Litorânea e Vale do Rio Guaribas, nas quais se encontram as cidades de Parnaíba e Picos, respectivamente. Este dado reflete uma concentração da assistência nestes locais.

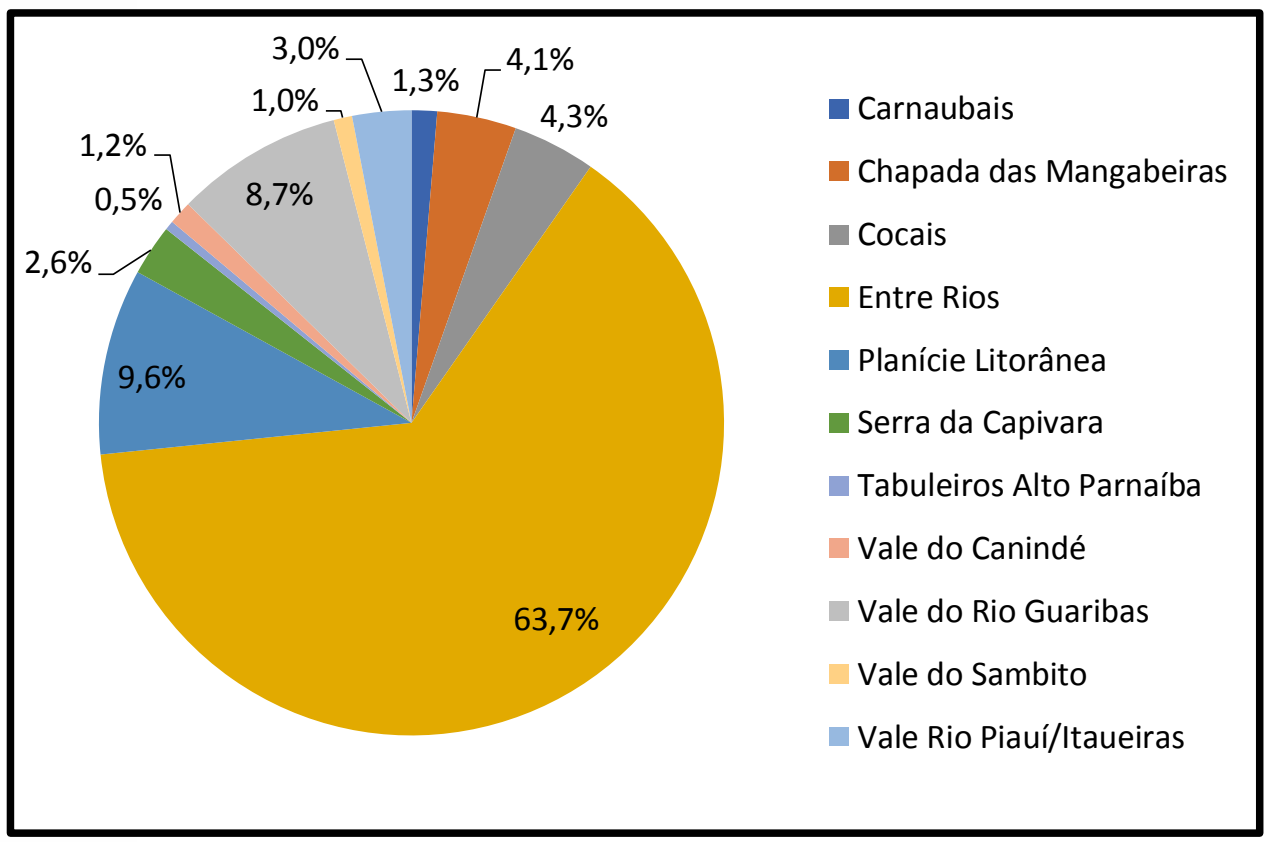

Gráfico 1 - Percentual de mortalidade infantil nos Territórios de Desenvolvimento do estado do Piauí por ocorrência do óbito. 2016, Teresina-PI. Fonte: Autores, dados DATASUS/TABNET.

Embora os óbitos ocorram principalmente em cidades populosas, os percentuais variam drasticamente quando analisados por residência da mãe (gráfico 2). O que se observa é uma disposição espacial do problema, abrangente a todo o estado. O TD Cocais passa a ser o segundo de maior percentual de óbito infantil mantendo-se praticamente estável os Territórios de Desenvolvimento anteriormente citados como as mais populosos. Os dados evidenciam um grande percentual de ncaminhamentos para a capital do Estado o que pode comprometer o atendimento e ssistência, caso não houvesse previsão para recebimento destes paciente 


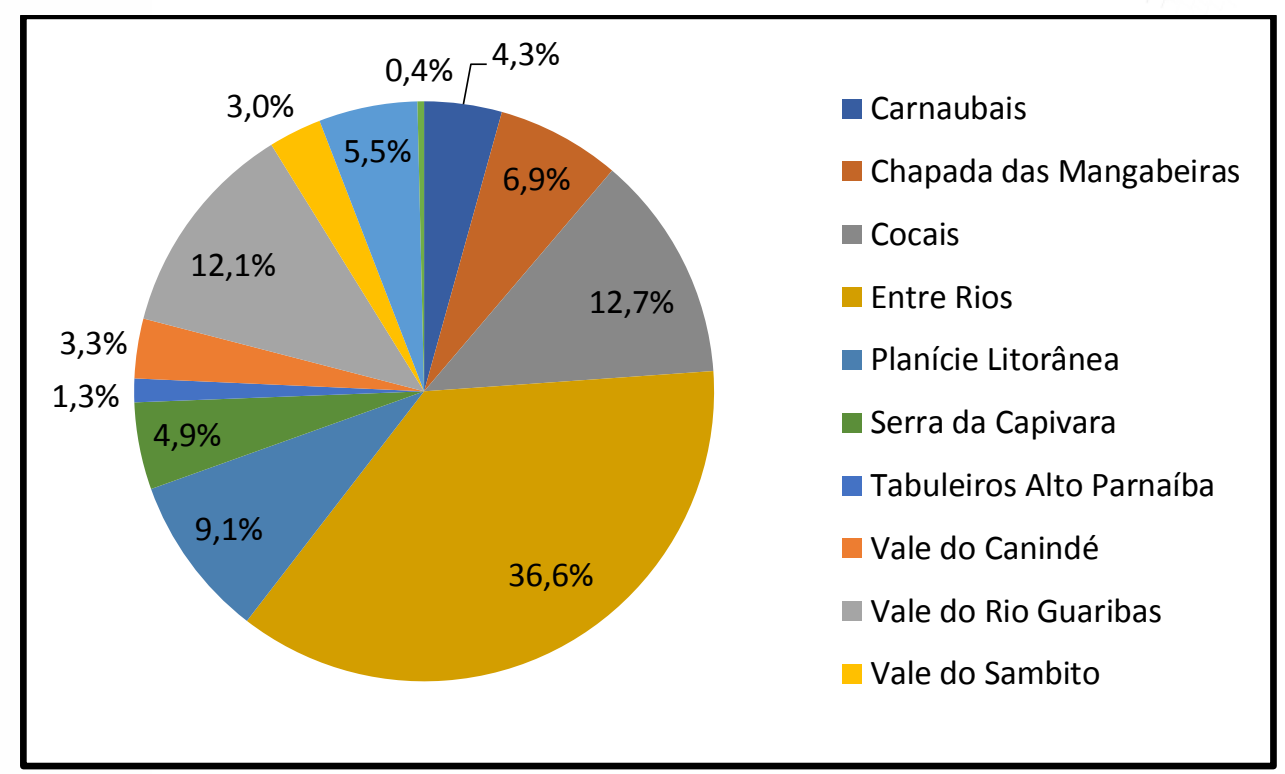

Gráfico 2 - Percentual de mortalidade infantil nos Territórios de Desenvolvimento do estado do Piauí por residência da mãe. Teresina-PI, 2016.

Fonte: Autores, dados DATASUS/TABNET.

Observou-se uma queda do número absoluto de óbitos entre os anos de 2007 e 2014, com moderada estabilização entre os anos 2004 e 2005, e um razoável aumento entre 2015 e 2016. Apesar das oscilações a curva desenhada matematicamente (em vermelho), mostra uma tendência à redução do número absoluto de óbito nos anos estudados.

Analisando a tabela 1, pode-se notar que, em quase todas os TD, a mortalidade neonatal precoce compreendeu mais de $2 / 3$ dos óbitos neonatais, sendo o pior resultado o da Território Vale do Rio Guaribas (com quase 71\% dos óbitos infantis na idade neonatal precoce) e o melhor, Vale do Canindé (com seus 46,5\%). Tais percentuais são de relevância pois refletem a atenção à saúde de cada $T D$, o acompanhamento pré-natal e o pós-natal

Um alto percentual de morte neonatal somado ao alto percentual de morte neonatal precoce dos Territórios do estado do Piauí, demonstram a falta de adequação dos serviços de saúde oferecidos às gestantes e as parturientes em relação às necessidades das mesmas. Contrapondo a isso o TD Entre Rios, apesar do maior número absoluto de óbitos infantis, possui o segundo menor percentual de óbito eonatal precoce dentre os TD do estado, demonstrando uma assistência perinatal de hor qualidade em relação aos demais. 
Tabela 1 - Mortalidade neonatal precoce e tardia. Teresina-PI, 2016

\begin{tabular}{lcc}
\hline TERRITÓRIO DE & $\begin{array}{c}\text { \% NEONATAL } \\
\text { PESENVOLVIMENTO } \\
\text { PRECOCE }\end{array}$ & $\begin{array}{c}\text { \% NEONATAL } \\
\text { TARDIO }\end{array}$ \\
\hline Carnaubais & 61,29 & 4,03 \\
Chapada das & 67,36 & 5,96 \\
Mangabeiras & 62,96 & 8,89 \\
Cocais & 49,88 & 20,82 \\
Entre Rios & 68,10 & 12,36 \\
Planície Litorânea & 70,97 & 4,44 \\
Serra da Capivara & 62,22 & 2,22 \\
Tabuleiros Alto Parnaíba & 46,49 & 14,91 \\
Vale do Canindé & 73,16 & 7,60 \\
Vale do Rio Guaribas & 61,11 & 4,44 \\
Vale do Sambito & 64,81 & 5,57 \\
Vale Rio Piauí/Itaueiras & 56,22 & 16,30 \\
\hline Total &
\end{tabular}

Fonte: Autores, dados DATASUS/TABNET.

Identificou-se, ainda, que mais de $53 \%$ dos óbitos são de RN de baixo peso, ou seja, que nasceram com menos de 2500g (Tabela 2). Este valor não reflete a realidade observada por estudo ${ }^{22}$ realizado na região centro-oeste do país o qual encontrou um percentual muito superior de óbitos entre $\mathrm{RN}$ de baixo peso. Os dados obtidos para o Piauí refletem mais uma baixa qualidade ao seguimento pré-natal, natal e pós-natal, que uma possível carência nutricional, apesar do número grande de $\mathrm{RN}$ com baixo peso ao nascer.

Observou-se também um maior percentual de óbitos de RN com baixo peso ao nascer no TD Entre Rios, fato justificado por se tratar de uma região referência no atendimento a qual, geralmente, recebe os casos mais complicados encaminhados de outras cidades.

O número de RN com baixo peso ao nascer podem ser justificados em parte pelos nascimentos prematuros. Notou-se que quase $50 \%$ da totalidade dos óbitos nfantis registrados no estado no período da pesquisa são referentes a $\mathrm{RN}$ com menos 37 semanas de idade gestacional. Mais uma vez os percentuais observados nos andes centros são superiores aos observados nas demais localidades justificado pelo el de complicações dos casos que chegam a estas localidades. 
Estes valores revelam, ainda, que a maior parte dos óbitos são de $\mathrm{RN}$ a termo e dentro do peso esperado ao nascer, demonstrando carência assistencial principalmente nas primeiras horas de vida quando se associa esta informação aos grandes índices de morte neonatal precoce, conforme Tabela 2.

Tabela 2 - Mortalidade infantil segundo idade gestacional. Teresina-PI, 2017

\begin{tabular}{|c|c|c|c|c|}
\hline $\begin{array}{l}\text { REGIÃO DE SAÚDE } \\
\text { (CIR) }\end{array}$ & $\begin{array}{c}\text { PRÉ- } \\
\text { TERMO } \\
\%\end{array}$ & $\begin{array}{c}\text { A } \\
\text { TERMO } \\
\%\end{array}$ & $\begin{array}{c}\text { PÓS- } \\
\text { TERMO } \\
\%\end{array}$ & $\begin{array}{c}\text { IGNORADO } \\
\%\end{array}$ \\
\hline Carnaubais & 28,23 & 50,00 & 0,81 & 20,97 \\
\hline \multicolumn{5}{|l|}{ Chapada das } \\
\hline Mangabeiras & 35,23 & 42,75 & 3,11 & 18,91 \\
\hline Cocais & 27,16 & 45,93 & 2,47 & 24,44 \\
\hline Entre Rios & 55,97 & 26,61 & 0,83 & 16,58 \\
\hline Planície Litorânea & 43,16 & 31,35 & 1,88 & 23,62 \\
\hline Serra da Capivara & 36,29 & 39,52 & 0,81 & 23,39 \\
\hline Tabuleiros Alto Parnaíba & 31,11 & 37,78 & 2,22 & 28,89 \\
\hline Vale do Canindé & 21,93 & 45,61 & 0,88 & 31,58 \\
\hline Vale do Rio Guaribas & 39,09 & 33,70 & 1,72 & 25,49 \\
\hline Vale do Sambito & 31,11 & 43,33 & 0,00 & 25,56 \\
\hline Vale Rio Piauí/Itaueiras & 29,62 & 37,98 & 3,14 & 29,27 \\
\hline Total & 48,73 & 30,61 & 1,24 & 19,42 \\
\hline
\end{tabular}

Fonte: Autores, dados DATASUS/TABNET

Outra informação relevante extraída da tabela 2 é o alto número de RN com idade gestacional ignorada o que pode estar relacionado a carência assistencial durante o período gestacional, ou seja, um pré-natal de qualidade questionável.

As cidades com maiores números de óbitos observados foram Teresina, Parnaíba e Picos, tanto em relação a ocorrência, como residência da mãe. Quando se observa os óbitos por residência da mãe o que se nota é um acentuado número de óbitos em cidades de médio porte como Miguel Alves, Barras, União, cidades estas que não aparecem entre as dez com maior número absoluto de óbitos por ocorrência. Este enquadramento pode orientar uma investigação da qualidade assistencial nestes focos, principalmente no que tange ao acompanhamento pré-natal.

Dentre as causas de mortalidade infantil no estado do Piauí, quase $64 \%$ estão assificadas no grupo XVI do Código Internacional de Doenças (CID) e referem-se a cções originadas no período perinatal, ou seja, a maior parte dos óbitos infantis rridos no estado ainda tem sido relacionados a causas evitáveis. Tais afecções 
poderiam ser evitadas por meio de melhoria na assistência pré-natal e estratégias voltadas a educação em saúde. É entendido, entretanto, a impossibilidade de zerar a mortalidade infantil, pois algumas causas não são evitáveis como parte do grupo XVII.

Quadro 1 - Principais grupos de causas de óbito infantil e neonatal, entre os anos de 2007 a 2016, no estado do Piauí. Teresina-PI, 2017

\begin{tabular}{|l|c|c|}
\hline GRUPO CID & N ÓBITOS INFANTIS $^{0}$ & 63,96 \\
\hline $\begin{array}{l}\text { XVI - Algumas afecções } \\
\text { originadas no período } \\
\text { perinatal }\end{array}$ & 6022 & 18,85 \\
\hline $\begin{array}{l}\text { XVII - Malformações } \\
\text { congênita, deformidades } \\
\text { enormalidades } \\
\text { congênitas }\end{array}$ & 1775 & 4,50 \\
\hline $\begin{array}{l}\text { I Algumas doenças } \\
\text { infecciosas parasitárias }\end{array}$ & 424 & 4,24 \\
\hline $\begin{array}{l}\text { X - Doenças do aparelho } \\
\text { respiratório }\end{array}$ & 399 & 2,24 \\
\hline $\begin{array}{l}\text { XVIII - Sintomas, sinais e } \\
\text { achados anormais } \\
\text { de exame clínico e } \\
\text { laboratorial, não } \\
\text { classificado em outra } \\
\text { parte }\end{array}$ & 211 & \\
\hline
\end{tabular}

Fonte: Autores, dados DATASUS/TABNET

O Quadro 01 apresenta as principais grupos de causas de óbito infantil e neonatal, entre os anos de 2007 a 2016, no estado do Piauí ratificando a situação de que muitas mortes poderiam ser evitadas com uma boa assistência ao pré-natal e ao parto e puerpério.

\section{Conclusões}

O presente estudo evidenciou uma concentração do atendimento nas regiões mais populosas do estado, bem como um maior número de óbitos nestas mesmas regiões. Percebeu-se que quanto maior a adesão pelo correto preenchimento das clarações de óbito e declaração de nascido vivo, melhor a qualidade da informação ada a partir dos dados disponibilizados no DATASUS. 
Além disso, ficou constatado que, apesar dos esforços na diminuição do número de óbitos infantis nos últimos anos, ainda ocorrem muitos óbitos por causas evitáveis. Estratégias devem ser apresentadas para melhor atender aos anseios da população, seja um redirecionamento de recursos, capacitação dos profissionais do atendimento básico de maneira mais incisiva, educação da população alvo (gestantes) por meio de palestras e orientações pela equipe multiprofissional.

Os achados revelaram as principais características dos óbitos neonatais no Piauí na série temporal (2007-2016), as quais permitem direcionar e orientar novas estratégias para a resolução da atual situação de óbitos, tais como ações adequadas, facilidade no acesso aos serviços de saúde, com oferta de assistência de qualidade e que contemple todas as fases da mulher e da criança.

\section{Referências}

1. Brasil. Secretaria de Atenção à Saúde. Departamento de Atenção Básica. Pacto nacional pela redução da mortalidade materna e neonatal. 2004.

2. United Nations. Transforming our world: the 2030 Agenda for Sustainable Development. 2015.

3. Brasil. Secretaria de Atenção à Saúde. Departamento de atenção. Atenção ao prénatal de baixo risco. Brasília: Ministério da Saúde; 2012

4. Brasil. Secretaria de Atenção à Saúde. Departamento de ações prognósticas estratégicas. Área técnica de saúde da mulher. Pré-natal e puerpério: atenção qualificada e humanizada - manual técnico. Brasília: Ministério da Saúde; 2005.

5. Warmiling CM, Farjado AP, Meyer DE, Bedos C. Práticas sociais de medicalização \& humanização no cuidado de mulheres na gestação. Cad. Saúde Pública, 2018;34(4) e00009917.

6. Viellas EF, Domingues SMM, Dias MAB, Gama SGN, Filha MMT, Costa JV, et al. Assistência pré-natal no Brasil. Cad. Saúde Pública. 2014;30:85-100.

Tamasi E, Fernandes PAA, Fischer T, Siqueira FCV, Silveira DS, Thume E, et al. ualidade da atenção pré-natal na rede básica de saúde do Brasil: indicadores e esigualdades sociais. Cad. Saúde Pública. 2017;33(3)e00195815.

Ferraz L, Bordignon M. Mortalidade materna no Brasil: uma realidade que precisa horar. Rev. baiana saúde pública. 2012;36(2). 
9. Sousa DMN, Mendes IC, Oliveira ET, Chagas ACMA, Catunda HLO, Oria MOB. Mortalidade materna por causas hipertensivas e hemorrágicas: uma análise epidemiológica de uma década. Rev. Enferm. UERJ. 2014;22(4):500-506.

10. Brasil. Ministério da Saúde. Secretaria de Vigilância em Saúde. Secretaria de Atenção à Saúde. Manual de vigilância do óbito infantil e fetal e do Comitê de Prevenção do Óbito Infantil e Fetal. 2. ed. Brasília : Ministério da Saúde; 2009.

11. Guimarães WSG, Parente RCP, Guimarães TLF, Garleno L. Acesso e qualidade da atenção pré-natal na Estratégia Saúde da Família: infraestrutura, cuidado e gestão. Cad. Saúde Pública. 2018;34(5).

12. Rasella D, Basu S, Hone T, Paes-Sousa R, Ockê-Reis CO, Millett C. Child morbidity and mortality associated with alternative policy responses to the economic crisis in Brasil: A nationwide microsimulation study. PLOS Med. 2018;15(5).

13. Silva SBIM, Serbena IA, Bastos RMR. Correlação entre a expansão da estratégia de Saúde da Família e a Taxa de Mortalidade Infantil no Brasil, de 2008 a 2014. Rev. APS. 2016;19(2):338-339.

14. Pinheiro JMF, Tinoco LS, Rocha ASS, Rodrigues MP, Lyra CO, Ferreira MAF. Atenção à criança no período neonatal: avaliação do pacto de redução da mortalidade neonatal no Rio Grande do Norte, Brasil. Ciênc. Saúde Colet. 2016;21(1):243-252.

15. Lansky S, Friche AAL, Silva AAM, Campos D, Bittencourt SDA, Carvalho ML, et al. Pesquisa nascer no Brasil: perfil da mortalidade neonatal e avaliação da assistência à gestante e ao recém-nascido. Cad. Saúde Pública. 2014;30(1):192-207.

16. França EB, Lansky S, Rego MAS, Malta DC, França JS, Teixeira R, et al. Principais causas da mortalidade na infância no Brasil, em 1990 e 2015: estivamtivas do estudo de Carga Global de Doença. Rev. Bras. Epidemiol. 2017;20(1):46-60.

17. Lucena L, Bocalon Cagliari GH, Tanaka J, Bonamigo EL. Declaração de óbito: preenchimento pelo corpo clínico de um hospital universitário. Revista bioét. 2014;22(2):318-324.

18. Gabriel GP, Chiquetto L, Morcillo AM, Ferreira MC, Bazan IGM, Daolio LD, et al. Avaliação das informações das Declarações de Nascidos Vivos do Sistema de Informação sobre Nascidos Vivos (Sinasc) em Campinas. Rev. Paul. Pediatria. 2014;32(3):183-188.

19. Costa MFS, Junior SCG, Magluta C. Análise da distribuição dos nascimentos com arcadores de gravidade em maternidades com unidade de terapia intensiva neonatal Sistema Único de Saúde. Cad. Saúde Coletiva. 2018;26(2):125-130. 
20. Nascimento SG, Oliveira CM, Sposito V, Ferreira DK, Bonfim CV. Mortalidade infantil por causas evitáveis em uma cidade do Nordeste do Brasil. Rev. bras. enferm. [online]. 2014;67(2):208-212.

21. Rede Interagencial de Informação para a Saúde. Indicadores básicos para a saúde no Brasil: conceitos e aplicações. 2. ed. Brasília: Organização Pan-Americana da Saúde; 2008.

22. Gaiva MAM, Fujimori E, Sato AP. Mortalidade Neonatal: análise das causas evitáveis. Rev. Enferm. UERJ. 2015;23(2):247-253. 